\title{
Inflaton perturbations in brane-world cosmology with induced gravity
}

\author{
Kazuya Koyama ${ }^{1}$ and Shuntaro Mizuno ${ }^{2}$ \\ ${ }^{1}$ Institute of Cosmology \& Gravitation, University of Portsmouth, Portsmouth PO1 2EG, United Kingdom \\ 2 Research Center for the Early Universe (RESCEU), \\ Graduate School of Science, The University of Tokyo, Tokyo 113-0033, Japan
}

\begin{abstract}
We study cosmological perturbations in the brane models with an induced Einstein-Hilbert term on a brane. We consider an inflaton confined to a de Sitter brane in a five-dimensional Minkowski spacetime. Inflaton fluctuations excite Kaluza-Klein modes of bulk metric perturbations with mass $m^{2}=-2(2 \ell-1)(\ell+1) H^{2}$ and $m^{2}=-2 \ell(2 \ell+3) H^{2}$ where $\ell$ is an integer. There are two branches ( \pm branches) of solutions for the background spacetime. In the + branch, which includes the selfaccelerating universe, a resonance appears for a mode with $m^{2}=2 H^{2}$ due to a spin-0 perturbation with $m^{2}=2 H^{2}$. The self-accelerating universe has a distinct feature because there is also a helicity0 mode of spin-2 perturbations with $m^{2}=2 H^{2}$. In the - branch, which can be thought as the Randall-Sundrum type brane-world with the high energy quantum corrections, there is no resonance. At high energies, we analytically confirm that four-dimensional Einstein gravity is recovered, which is related to the disappearance of van Dam-Veltman-Zakharov discontinuity in de Sitter spacetime. On sufficiently small scales, we confirm that the lineariaed gravity on the brane is well described by the Brans-Dicke theory with $\omega=3 H r_{c}$ in - branch and $\omega=-3 H r_{c}$ in + branch, respectively, which confirms the existence of the ghost in + branch. We also study large scale perturbations. In + branch, the resonance induces a non-trivial anisotropic stress on the brane via the projection of Weyl tensor in the bulk, but no instability is shown to exist on the brane.
\end{abstract}

PACS numbers:

\section{INTRODUCTION}

There has been tremendous interest over the last several years in the brane-world scenario where we are assumed to be living on a four-dimensional hypersurface (brane) in a higher-dimensional spacetime (bulk) [1]. The simplest example of this scenario is proposed by Randall and Sundrum where there is a brane embedded in a five-dimensional anti-de Sitter (AdS) spacetime [2]. They showed that the four-dimensional gravity is recovered at low energies, because gravity is confined to a single positive-tension brane even if the extra dimension is not compact. Therefore, in order to see the deviation from the conventional four-dimensional gravity, it is necessary to investigate the phenomena occurred at high energies.

One of the most promising tools to extract the information of the extra-dimension is the primordial density fluctuations generated in the period of inflation in the early universe. In the inflation model where inflation is driven by an inflaton field confined to the brane, the amplitude of the curvature perturbation is calculated in the extremely slow-roll limit where the coupling between inflaton field fluctuation and bulk perturbations can be neglected [3]. This work is proceeded to go beyond the zero-th order slow-roll approximation by solving the bulk metric perturbations classically [4, [5]. It is shown that we could have significant effects from the back-reaction due to the coupling to five-dimensional gravitational perturbations. On the other hand, it was pointed out that the localized matter on a brane can induce gravity on the brane via quantum loop corrections at high energies [6]. This induced gravity can act as the ultra-violet cut-off for the inflaton perturbations.

Based on the induced gravity scenario, Dvali, Gabadadze, and Porrati (DGP) proposed a brane-world model with induced gravity [] in which the four-dimensional brane is embedded in a five-dimensional Minkowski spacetime. (For a review of the phenomenology of DGP model, see [8].) In the presence of the induced gravity, according to the embedding of the brane in the bulk, there appear two branches of background solutions. In + branch, the size of the four-dimensional hypersurface becomes minimum at the brane while, in - branch, it becomes maximum at the brane. Inflation models in this scenario were studied in [9, 10, 11].

In this paper, we study the behavior of five-dimensional metric perturbations excited by the inflaton perturbations confined to the brane in DGP model. The solutions in - branch are regarded as the high energy limit of the RandallSundrum model including the curvature corrections on a brane, depending on the parameters. Then our analysis can be used to discuss the generation of primordial fluctuations in these models. The solutions in + branch are largely used to explain the present cosmic acceleration without introducing dark energy in the DGP model [12], but it turns out that they are plagued by the ghost instability [13, 14, 15, 16, 17]. However, the study of the gravitational property of this model in the presence of matter and cosmic expansion is still very limited [18, 19, 20] so it is also worth considering the solutions in this branch.

The structure of the rest of the paper is as follows. In section II, we derive the basic equations for the five- 
dimensional metric perturbation and the boundary condition imposed at the brane and then summarize the solution for the vacuum brane obtained in [15]. In section III, we provide the solutions for curvature perturbations on the brane in the presence of the scalar field on the brane. Next we discuss the appearance and disappearance of van DamVeltman-Zakharov discontinuity in section IV. In section V we show the comparison of the behavior of perturbations on small scales with the Brans-Dicke theory. The solutions for perturbations on large scales are discussed in section VI. Section VII is devoted to conclusions. Technical details of the calculation of the $m^{2}=2 H^{2}$ mode and the brief summary of the cosmological perturbation in four-dimensional Brans-Dicke gravity are presented in the Appendix A and $\mathrm{B}$, respectively.

\section{BULK GRAVITONS WITH A DE SITTER BRANE}

\section{A. Background cosmology}

We consider a four-dimensional brane-world model with a five-dimensional infinite-volume bulk. The action is given by

$$
S=\frac{1}{2 \kappa^{2}} \int d^{5} x \sqrt{-g}^{(5)} R+\int d^{4} x \sqrt{-\gamma}\left[\frac{1}{\kappa^{2}} K+L_{\text {brane }}+\frac{1}{2 \kappa_{4}^{2}} R\right]
$$

where $\kappa^{2}$ is the fundamental five-dimensional gravitational constant, $K$ is the trace of extrinsic curvature $K_{A B}$ on the brane and $L_{\text {brane }}$ is a Lagrangian for brane-localized matter. In addition, we consider the last term, an intrinsic curvature term on the brane which plays a crucial role in this model. Here, $\kappa_{4}^{2}=8 \pi G$ is the four-dimensional gravitational constant. We define a crossover scale $r_{c}$ as

$$
r_{c}=\frac{\kappa^{2}}{2 \kappa_{4}^{2}}
$$

We are interested in the gravitational property of this model in a cosmological setting. By assuming the flat Friedmann-Robertson-Walker metric on the brane and neglecting the effect from the five-dimensional Weyl tensor in the background, the following Friedmann like equation is derived [12];

$$
H^{2}-\frac{\epsilon}{r_{c}} H=\frac{\kappa_{4}^{2}}{3} \rho,
$$

where $H$ is a Hubble parameter on the brane and $\epsilon= \pm 1$, which is the parameter related to the embedding of the brane in the bulk. We call the case with $\epsilon=1+$ branch, while $\epsilon=-1-$ branch.

In this paper, we restrict ourselves to de Sitter background $H=$ constant. In this case, the energy density is constant and given by

$$
\rho=\frac{3 H}{\kappa_{4}^{2} r_{c}}\left(r_{c} H-\epsilon\right)
$$

The 5D solution for the metric with the four-dimensional de Sitter brane can be obtained as

$$
d s^{2}=d y^{2}+N(y)^{2} \gamma_{\mu \nu} d x^{\mu} d x^{\nu}, \quad N(y)=1+\epsilon H y,
$$

where $\gamma_{\mu \nu}$ is the metric for the de Sitter spacetime and the brane is located at $y=0$. During a slow-roll inflation, the Hubble scale is varying slowly, thus we can approximate the background geometry by (5).

\section{B. Master variable for perturbations in the Minkowski bulk}

Now let us consider the scalar perturbation. In order to solve the perturbations in this background, it is convenient to use five-dimensional longitudinal gauge, given by

$$
d s^{2}=\left(1+2 A_{y y}\right) d y^{2}+2 N(y) A_{y} d y d t+N(y)^{2}\left[-(1+2 A) d t^{2}+a(t)^{2}(1+2 \mathcal{R}) \delta_{i j} d x^{i} d x^{j}\right],
$$

where $a(t)=\exp (H t)$. 
In the absence of bulk matter perturbations, five-dimensional perturbed Einstein equations ${ }^{(5)} \delta G^{A}{ }_{B}=0$ are solved in a Minkowski background if the metric perturbations are derived from a 'master variable', $\Omega$ [21] (see also [22]);

$$
\begin{aligned}
A & =-\frac{1}{6 a N}\left(2 \Omega^{\prime \prime}-\frac{N^{\prime}}{N} \Omega^{\prime}+\frac{1}{N^{2}} \ddot{\Omega}\right), \\
A_{y} & =\frac{1}{a N^{2}}\left(\dot{\Omega}^{\prime}-\frac{N^{\prime}}{N} \dot{\Omega}\right), \\
A_{y y} & =\frac{1}{6 a N}\left(\Omega^{\prime \prime}-2 \frac{N^{\prime}}{N} \Omega^{\prime}+\frac{2}{N^{2}} \ddot{\Omega}\right), \\
\mathcal{R} & =\frac{1}{6 a N}\left(\Omega^{\prime \prime}+\frac{N^{\prime}}{N} \Omega^{\prime}-\frac{1}{N^{2}} \ddot{\Omega}\right),
\end{aligned}
$$

where dot and prime denote derivatives with respect to $t$ and $y$, respectively. The perturbed five-dimensional Einstein equations yield a single wave equation governing the evolution of the master variable $\Omega$ in the bulk:

$$
\ddot{\Omega}-3 H \dot{\Omega}-\left(\Omega^{\prime \prime}-2 \frac{N^{\prime}}{N} \Omega^{\prime}\right)+\frac{k^{2}}{a^{2}} \Omega=0 .
$$

Solutions for the master equation can be separated into eigenmodes of the time-dependent equation on the brane and bulk mode equation:

$$
\Omega(t, y ; \vec{x})=\int d^{3} \vec{k} d m g_{m}(t) f_{m}(y) e^{i k x},
$$

where

$$
\begin{aligned}
& \ddot{g}_{m}-3 H \dot{g}_{m}+\left[m^{2}+\frac{k^{2}}{a^{2}}\right] g_{m}=0, \\
& f_{m}^{\prime \prime}-2 \frac{N^{\prime}}{N} f_{m}^{\prime}+\frac{m^{2}}{N^{2}} f_{m}=0 .
\end{aligned}
$$

\section{Boundary condition for $\Omega$}

In order to solve $\Omega$, we must specify the boundary condition for $\Omega$ from the junction conditions at the brane. The junction conditions in this model have been found already in literatures (see [23, 24]), but, we re-derive these so that the property of linearized gravity is transparent.

For simplicity, we consider the case where the anisotropic stress of the matter on the brane does not exist. There are two important contributions to the junction conditions. One is that, from Eq. (83), we must take into account not only the matter energy momentum, but also the contribution from the induced gravity on the brane. The other is that we should take into account the brane bending $\xi$ [25, 26]. The metric perturbations in five-dimensional longitudinal gauge evaluated at $y=0$ is not the induced metric perturbations on the brane. This is due to the anisotropic stress coming from the induced gravity. The junction conditions are given by

$$
\begin{aligned}
& \frac{1}{a}[\ddot{\mathcal{F}}+2 H \dot{\mathcal{F}}]-2 \ddot{\xi}-4 H \dot{\xi}+6 H^{2} \xi-\frac{4}{3} \frac{k^{2}}{a^{2}} \xi=\kappa^{2}\left(\delta p_{\mathrm{m}}+\delta p_{\mathrm{g}}\right), \\
& -\frac{1}{a} \dot{\mathcal{F}}+2 \dot{\xi}-2 H \xi=\kappa^{2}\left(\delta q_{\mathrm{m}}+\delta q_{\mathrm{g}}\right), \\
& -\frac{1}{a}\left[3 H \dot{\mathcal{F}}+\frac{k^{2}}{a^{2}} \mathcal{F}\right]+6 H \dot{\xi}-6 H^{2} \xi+2 \frac{k^{2}}{a^{2}} \xi=\kappa^{2}\left(\delta \rho_{\mathrm{m}}+\delta \rho_{\mathrm{g}}\right),
\end{aligned}
$$

where

$$
\mathcal{F}=\Omega^{\prime}-\epsilon H \Omega
$$

Here the brane bending mode is given by

$$
\xi=-r_{c}\left(A_{(b)}+\mathcal{R}_{(b)}\right)
$$


where $A_{(b)}$ and $\mathcal{R}_{(b)}$ are the induced metric perturbations on the brane that are related with the metric perturbations in five-dimensional longitudinal gauge as

$$
\begin{aligned}
A_{(b)} & =\frac{1}{1-2 \epsilon H r_{c}}\left[\left(1-\epsilon H r_{c}\right) A+\epsilon H r_{c} \mathcal{R}\right], \\
\mathcal{R}_{(b)} & =\frac{1}{1-2 \epsilon H r_{c}}\left[\left(1-\epsilon H r_{c}\right) \mathcal{R}+\epsilon H r_{c} A\right],
\end{aligned}
$$

where $\mathcal{R}$ and $A$ are metric perturbations in five-dimensional longitudinal gauge evaluated at $y=0$, which can be expressed by $\Omega$ as

$$
\begin{aligned}
\mathcal{R} & =\frac{1}{6 a}\left[3 \epsilon H \mathcal{F}-3 H(\dot{\Omega}-H \Omega)+\frac{k^{2}}{a^{2}} \Omega\right], \\
A & =\frac{1}{6 a}\left[-3 \epsilon H \mathcal{F}-3 \ddot{\Omega}+6 H \dot{\Omega}-3 H^{2} \Omega-2 \frac{k^{2}}{a^{2}} \Omega\right] .
\end{aligned}
$$

$\delta \rho_{m}, \delta p_{m}$ and $\delta q_{m}$ are the components of the perturbed energy momentum tensor of the matter on the brane and

$$
\begin{aligned}
& \delta p_{\mathrm{g}}=\frac{2}{\kappa_{4}^{2}}\left[-3 H^{2} A_{(b)}-H \dot{A}_{(b)}+\ddot{\mathcal{R}}_{(b)}+3 H \dot{\mathcal{R}}_{(b)}+\frac{k^{2}}{3 a^{2}}\left(A_{(b)}+\mathcal{R}_{(b)}\right)\right] \\
& \delta q_{\mathrm{g}}=\frac{2}{\kappa_{4}^{2}}\left(H A_{(b)}-\dot{\mathcal{R}}_{(b)}\right) \\
& \delta \rho_{\mathrm{g}}=\frac{2}{\kappa_{4}^{2}}\left[3 H\left(-\dot{\mathcal{R}}_{(b)}+H A_{(b)}\right)-\frac{k^{2}}{a^{2}} \mathcal{R}_{(b)}\right]
\end{aligned}
$$

are contributions from the induced gravity. If we do not take into account the induce gravity term which corresponds to the limit $r_{c} \rightarrow \infty$, the quantities $\xi, \delta p_{\mathrm{g}}, \delta q_{\mathrm{g}}$ and $\delta \rho_{\mathrm{g}}$ can be neglected and the junction conditions in Randall-Sundrum type brane-world [4, 23, 27] are reproduced.

Defining $\mathcal{G}$ as

$$
\mathcal{G}=\left(1-2 \epsilon H r_{c}\right) \mathcal{F}-r_{c}\left(2 H^{2}-m^{2}\right) \Omega,
$$

the junction conditions (12),(13), (14) are amazingly simplified as

$$
\begin{aligned}
& -3 H \dot{\mathcal{G}}-\frac{k^{2}}{a^{2}} \mathcal{G}=\kappa^{2} a \delta \rho_{\mathrm{m}}, \\
& -\dot{\mathcal{G}}=\kappa^{2} a \delta q_{\mathrm{m}}, \\
& \ddot{\mathcal{G}}+2 H \dot{\mathcal{G}}=\kappa^{2} a \delta p_{\mathrm{m}} .
\end{aligned}
$$

\section{Solutions for a vacuum brane}

Before discussing the case with the matter perturbations on the brane, we briefly summarize the case for a vacuum brane, $\delta T^{\mu}{ }_{\nu}=0$ considered in [15, 16]. We will only consider a positive tension brane which means $H r_{c}>1$ for + branch and $H r_{c}>0$ for - branch. The solutions obtained here will serve as homogeneous solutions when we include matter perturbations.

For the vacuum brane, the boundary conditions for $\Omega$ given by Eqs. (25), (26) and (27) reduce to a single boundary condition on the master variable,

$$
\mathcal{G}=0
$$

For both branches, we find a tower of continuous massive modes starting from $m^{2}=(9 / 4) H^{2}$, which are the KaluzaKlein (KK) modes of the spin-2 perturbations.

In + branch $(\epsilon=1)$, in addition to continuous modes, there are two discrete modes which are normalizable. One is the mode with $m^{2}=2 H^{2}$ which is the spin-0 perturbation, and the other is the mode with $m_{d}^{2}=H^{2}\left(3 H r_{c}-1\right)\left(H r_{c}\right)^{-2}$ which is the helicity-0 excitation of the spin-2 perturbations [28]. For $H r_{c}>1$, the mass $m_{d}^{2}$ is in the range $0<m_{d}^{2}<2 H^{2}$. If $H r_{c}=1$, the helicity-0 excitation of the spin-2 perturbation has mass $m_{d}^{2}=2 H^{2}$, which coincides with the mass of the spin- 0 perturbation. Then, there is a resonance between these two modes. It was also shown that the spin-2 helicity-0 mode becomes a ghost for $H r_{c}>1$. In Figure 1, we summarize the mass spectrum for $1 \leq H r_{c}$ in + branch. 


\section{Spectrum}

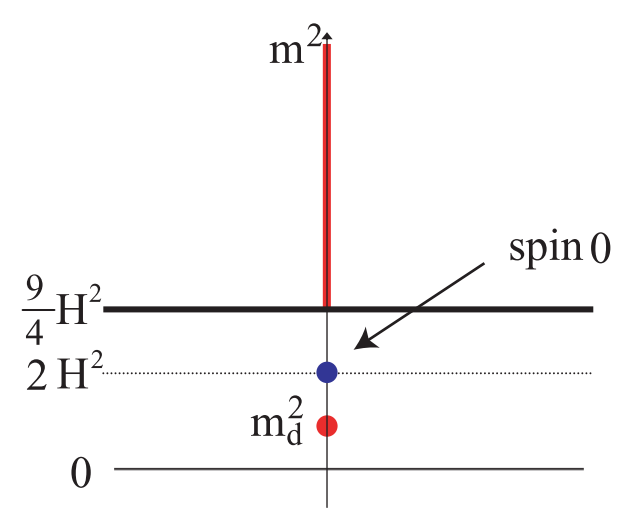

FIG. 1: Summary of the mass spectrum of the scalar perturbations in + branch [15]. Spin-2 perturbation has continuous modes with $m^{2} \geq(9 / 4) H^{2}$ and a discrete mode with $m^{2}=m_{d}^{2}$ while spin-0 perturbation has $m^{2}=2 H^{2}$. In the limit $H r_{c} \rightarrow 1$, both the helicity-0 excitation of spin- 2 perturbation and the spin- 0 perturbation have mass $m^{2}=2 H^{2}$ and there is a resonance [16].

\section{SCALAR FIELD ON THE BRANE}

\section{A. Bulk scalar modes}

Until now, we have not specified the matter on the brane. In the following, we model the matter on the brane as a canonical scalar field $\phi$ with potential $V(\phi)$ whose homogeneous part gives approximately de Sitter universe on the brane [4, 5].

In this case, the junction conditions (12), (13), (14) reduce to

$$
\begin{aligned}
& -3 H \dot{\mathcal{G}}-\frac{k^{2}}{a^{2}} \mathcal{G}=\kappa^{2} a\left[\dot{\phi} \dot{\delta \phi}+V,{ }_{\phi} \delta \phi\right], \\
& \dot{\mathcal{G}}=\kappa^{2} a \dot{\phi} \delta \phi, \\
& \ddot{\mathcal{G}}+2 H \dot{\mathcal{G}}=\kappa^{2} a\left[\dot{\phi} \dot{\delta \phi}-V,_{\phi} \delta \phi\right] .
\end{aligned}
$$

These equations can be thought as the boundary conditions for $\Omega$. Combining the junction conditions (29), (30), (31), we get an evolution equation for $\mathcal{G}$ :

$$
\ddot{\mathcal{G}}-\left(H+2 \frac{\ddot{\phi}}{\dot{\phi}}\right) \dot{\mathcal{G}}+\frac{k^{2}}{a^{2}} \mathcal{G}=0 .
$$

This gives the boundary condition for the time dependence of the master variable $\Omega$. Assuming that $\phi$ is slow-rolling, so that $|\ddot{\phi} / \dot{\phi}| \ll H$ in Eq. (32), which is valid for the de Sitter universe, the solution for $\mathcal{G}$ is

$$
\mathcal{G}=C_{1} \frac{\cos (-k \eta)}{-k \eta}+C_{2} \frac{\sin (-k \eta)}{-k \eta}
$$

We use the formulae for summation of Bessel functions,

$$
\begin{aligned}
& \sum_{\ell=0}^{\infty}(-1)^{\ell}\left(2 \ell+\frac{3}{2}\right) z^{-\frac{3}{2}} J_{2 \ell+\frac{3}{2}}(z)=\sqrt{\frac{1}{2 \pi}} \frac{\sin z}{z}, \\
& \sum_{l=0}^{\infty}(-1)^{\ell}\left(2 \ell+\frac{1}{2}\right) z^{-\frac{3}{2}} J_{2 \ell+\frac{1}{2}}(z)=\sqrt{\frac{1}{2 \pi}} \frac{\cos z}{z} .
\end{aligned}
$$

These show that an infinite sum of mode functions

$$
g_{m}=(-k \eta)^{-3 / 2} J_{\nu}(-k \eta), \quad \text { where } \nu^{2}=\frac{9}{4}-\frac{m^{2}}{H^{2}}
$$


can satisfy the boundary condition imposed on $\mathcal{G}$, where the spectrum of KK modes is given by

$$
\begin{aligned}
& \frac{m^{2}}{H^{2}}=-2(2 \ell-1)(\ell+1) \text { for } C_{1}, \\
& \frac{m^{2}}{H^{2}}=-2 \ell(2 \ell+3) \text { for } C_{2} .
\end{aligned}
$$

Figure 2 shows the mass spectrum of the scalar perturbation supported by the scalar field on the brane. Since there is a mode with $m^{2}=2 H^{2}$ regardless of the value of $H r_{c}$ in the + branch, the resonance inevitably appears. This gives qualitative difference between - branch and + branch. In addition, for $H r_{c}=1$, that is, on the self-accelerating universe, the spin-0 and spin- 2 modes are already degenerates. Thus the behavior of gravity is again qualitatively different from $H r_{c}>1$.

\section{Spectrum}

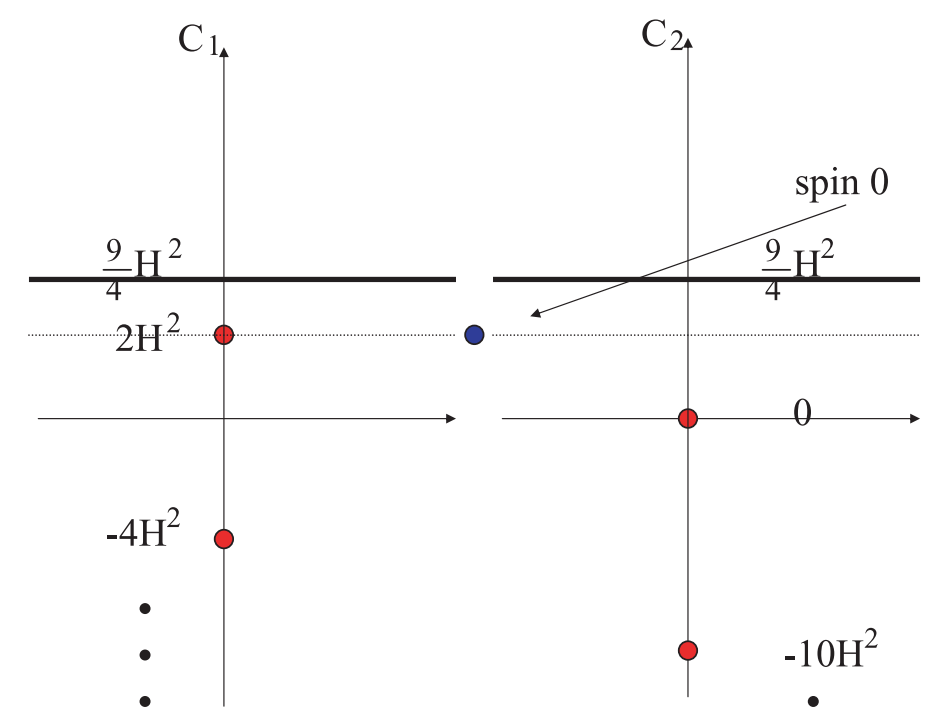

FIG. 2: Summary of the mass spectrum of the scalar perturbation sourced by the scalar field on the brane. For the modes $C_{1}$, there is a mode with $m^{2}=2 H^{2}$ regardless of the value of $H r_{c}$. Since there is another mode with $m^{2}=2 H^{2}$ by the spin-0 perturbation in the + branch, there is a resonance. In addition, for $H r_{c}=1$, the helicity-0 mode of spin- 2 perturbations also has $m^{2}=2 H^{2}$.

In order to examine the behavior of the master variable $\Omega$, we will consider - branch $(\epsilon=-1)$ and + branch $(\epsilon=1)$ separately in the following.

\section{$-\operatorname{branch}(\epsilon=-1)$}

Since there is a horizon at $y=1 / H$ in this branch, we should choose the solution in the $y$-direction so that the metric perturbations remain small as $y \rightarrow 1 / H$. By imposing the normalizability condition on $f_{m}$,

$$
\int_{0}^{1 / H} N(y)^{-4}\left|f_{m}(y)\right|^{2} d y<\infty, \quad \text { with } \quad N(y)=1-H y
$$

we obtain the solution for $\Omega$ in the bulk (for $y \geq 0$ ) as

$$
\begin{aligned}
\Omega(\eta, y)= & -C_{1} \frac{\sqrt{2 \pi}}{H} \sum_{\ell=0}^{\infty}(-1)^{\ell}\left(2 \ell+\frac{1}{2}\right) \frac{(1-H y)^{2+2 \ell}}{(2 \ell+1)\left\{2(\ell+1) H r_{c}+1\right\}}(-k \eta)^{-\frac{3}{2}} J_{2 \ell+\frac{1}{2}}(-k \eta) \\
& -C_{2} \frac{\sqrt{2 \pi}}{H} \sum_{\ell=0}^{\infty}(-1)^{\ell}\left(2 \ell+\frac{3}{2}\right) \frac{(1-H y)^{3+2 \ell}}{2(\ell+1)\left\{(2 \ell+3) H r_{c}+1\right\}}(-k \eta)^{-\frac{3}{2}} J_{2 \ell+\frac{3}{2}}(-k \eta) .
\end{aligned}
$$




\section{+ branch $(\epsilon=1)$}

In + branch, the bulk is infinite. We should choose the solution in the $y$-direction so that the metric perturbations remain small as $y \rightarrow \infty$ and the mode is normalizable for a single brane. We impose the normalizability condition on $f_{m}$ as

$$
\int_{0}^{\infty} N(y)^{-4}\left|f_{m}(y)\right|^{2} d y<\infty, \quad \text { with } \quad N(y)=1+H y
$$

Because of the existence of the resonance, we must treat the mode with $m^{2}=2 H^{2}$ separately. We also treat $H r_{c}=1$ case separately as the spin-2 and spin-0 degenerate (see Appendix A for derivations).

We obtain the solution for $\Omega$ in the bulk (for $y \geq 0$ ) as

$$
\begin{aligned}
\Omega(\eta, y)= & \Omega_{\left(m^{2}=2 H^{2}\right)}(\eta, y) \\
& -C_{1} \frac{\sqrt{2 \pi}}{H} \sum_{\ell=1}^{\infty}(-1)^{\ell}\left(2 \ell+\frac{1}{2}\right) \frac{(1+H y)^{1-2 \ell}}{2 \ell\left\{(2 \ell-1) H r_{c}+1\right\}}(-k \eta)^{-\frac{3}{2}} J_{2 \ell+\frac{1}{2}}(-k \eta) \\
- & C_{2} \frac{\sqrt{2 \pi}}{H} \sum_{\ell=0}^{\infty}(-1)^{\ell}\left(2 \ell+\frac{3}{2}\right) \frac{(1+H y)^{2 \ell}}{(2 \ell+1)\left(2 \ell H r_{c}+1\right)}(-k \eta)^{-\frac{3}{2}} J_{2 \ell+\frac{3}{2}}(-k \eta),
\end{aligned}
$$

where

$$
\begin{aligned}
\Omega_{\left(m^{2}=2 H^{2}\right)=} & C_{1} \frac{(1+H y)}{2 H\left(H r_{c}-1\right)}(-k \eta)^{-2}\{\alpha(-k \eta) \sin (-k \eta)+\beta(-k \eta) \cos (-k \eta)\} \\
& -C_{1} \frac{(1+H y) \ln (1+H y)}{H\left(H r_{c}-1\right)}(-k \eta)^{-2} \sin (-k \eta) \\
\alpha(-k \eta)= & \int^{-k \eta} d(-k \bar{\eta})(-k \bar{\eta})^{-2} \sin (-2 k \bar{\eta}) \\
\beta(-k \eta)= & \int^{-k \eta} d(-k \bar{\eta})(-k \bar{\eta})^{-2}\{\cos (-2 k \bar{\eta})-1\}
\end{aligned}
$$

for $H r_{c} \neq 1$ and

$$
\begin{aligned}
\Omega_{\left(m^{2}=2 H^{2}\right)=} & -\frac{C_{1}}{2 H}(1+H y)(-k \eta)^{-2}\{\tilde{\alpha}(-k \eta) \sin (-k \eta)+\tilde{\beta}(-k \eta) \cos (-k \eta)\} \\
& +\frac{C_{1}}{2 H}(1+H y) \ln (1+H y)(-k \eta)^{-2}\{\alpha(-k \eta) \sin (-k \eta)+\beta(-k \eta) \cos (-k \eta)\} \\
& -\frac{C_{1}}{2 H}(1+H y)\{\ln (1+H y)\}^{2}(-k \eta)^{-2} \sin (-k \eta), \\
\alpha(-k \eta)= & \int^{-k \eta} d(-k \bar{\eta})(-k \bar{\eta})^{-2} \sin (-2 k \bar{\eta}), \\
\beta(-k \eta)= & \int^{-k \eta} d(-k \bar{\eta})(-k \bar{\eta})^{-2}\{\cos (-2 k \bar{\eta})-1\}, \\
\tilde{\alpha}(-k \eta)= & \alpha(-k \eta)+\frac{1}{2} \int^{-k \eta} d(-k \bar{\eta})(-k \bar{\eta})^{-2}[\alpha(-k \bar{\eta}) \sin (-2 k \bar{\eta})+\beta(-k \bar{\eta})\{\cos (-2 k \bar{\eta})+1\}], \\
\tilde{\beta}(-k \eta)= & \beta(-k \eta)+\frac{1}{2} \int^{-k \eta} d(-k \bar{\eta})(-k \bar{\eta})^{-2}[\alpha(-k \bar{\eta})\{\cos (-2 k \bar{\eta})-1\}-\beta(-k \bar{\eta}) \sin (-2 k \bar{\eta})],
\end{aligned}
$$

for $H r_{c}=1$.

\section{B. Curvature perturbation on the brane (general solutions)}

Since we have obtained the general solutions for $\Omega$, from Eqs. (17), (18), (19) and (20) the induced metric perturbations on the brane can be obtained. We concentrate on the behavior of the curvature perturbation on the brane $\mathcal{R}_{(b)}$. As in the previous section, we will consider - branch $(\epsilon=-1)$ and + branch $(\epsilon=1)$ separately. 
- branch $(\epsilon=-1)$

In - branch $(\epsilon=-1)$, the curvature perturbation on the brane is given as

$$
\begin{aligned}
\mathcal{R}_{(b)}= & -\frac{\sqrt{2 \pi}}{6} \frac{C_{1}}{k} \frac{H^{2}}{\left(2 H r_{c}+1\right)} \sum_{\ell=0}^{\infty} \frac{(-1)^{\ell}\left(2 \ell+\frac{1}{2}\right)}{(2 \ell+1)\left\{2(\ell+1) H r_{c}+1\right\}} \\
& \times\left[6(\ell+1)(2 \ell+1) H r_{c}(-k \eta)^{-\frac{1}{2}} J_{2 \ell+\frac{1}{2}}(-k \eta)+2(2 \ell+1)(-k \eta)^{\frac{1}{2}} J_{2 \ell-\frac{1}{2}}(-k \eta)-(-k \eta)^{\frac{3}{2}} J_{2 \ell-\frac{3}{2}}(-k \eta)\right] \\
& -\frac{\sqrt{2 \pi}}{6} \frac{C_{2}}{k} \frac{H^{2}}{\left(2 H r_{c}+1\right)} \sum_{\ell=0}^{\infty} \frac{(-1)^{\ell}\left(2 \ell+\frac{3}{2}\right)}{2(\ell+1)\left\{(2 \ell+3) H r_{c}+1\right\}} \\
& \times\left[6(\ell+1)(2 \ell+3) H r_{c}(-k \eta)^{-\frac{1}{2}} J_{2 \ell+\frac{3}{2}}(-k \eta)+2(2 \ell+2)(-k \eta)^{\frac{1}{2}} J_{2 \ell+\frac{1}{2}}(-k \eta)-(-k \eta)^{\frac{3}{2}} J_{2 \ell-\frac{1}{2}}(-k \eta)\right] .
\end{aligned}
$$

$+\operatorname{branch}(\epsilon=1)$

In + branch $(\epsilon=1)$, the curvature perturbation on the brane is given as

$$
\begin{aligned}
\mathcal{R}_{(b)}= & \mathcal{R}_{(b)\left(m^{2}=2 H^{2}\right)} \\
& -\frac{\sqrt{2 \pi}}{6} \frac{C_{1}}{k} \frac{H^{2}}{\left(2 H r_{c}-1\right)} \sum_{\ell=1}^{\infty} \frac{(-1)^{\ell}\left(2 \ell+\frac{1}{2}\right)}{2 \ell\left\{(2 \ell-1) H r_{c}+1\right\}} \\
& \times\left[\left\{6 \ell(2 \ell-1) H r_{c}+12 \ell+3\right\}(-k \eta)^{-\frac{1}{2}} J_{2 \ell+\frac{1}{2}}(-k \eta)-2(2 \ell+1)(-k \eta)^{\frac{1}{2}} J_{2 \ell-\frac{1}{2}}(-k \eta)+(-k \eta)^{\frac{3}{2}} J_{2 \ell-\frac{3}{2}}(-k \eta)\right] \\
& -\frac{\sqrt{2 \pi}}{6} \frac{C_{2}}{k} \frac{H^{2}}{\left(2 H r_{c}-1\right)} \sum_{\ell=0}^{\infty} \frac{(-1)^{\ell}\left(2 \ell+\frac{3}{2}\right)}{(2 \ell+1)\left\{2 \ell H r_{c}+1\right\}} \\
& \times\left[\left\{6 \ell(2 \ell+1) H r_{c}+12 \ell+9\right\}(-k \eta)^{-\frac{1}{2}} J_{2 \ell+\frac{3}{2}}(-k \eta)-4(\ell+1)(-k \eta)^{\frac{1}{2}} J_{2 \ell+\frac{1}{2}}(-k \eta)+(-k \eta)^{\frac{3}{2}} J_{2 \ell-\frac{1}{2}}(-k \eta)\right],
\end{aligned}
$$

where

$$
\begin{aligned}
\mathcal{R}_{(b)\left(m^{2}=2 H^{2}\right)}= & -\frac{C_{1}}{6 k} \frac{H^{2}}{\left(2 H r_{c}-1\right)\left(H r_{c}-1\right)}\left[3\left(H r_{c}-1\right)(-k \eta)^{-1} \sin (-k \eta)\right. \\
& +\frac{\alpha(-k \eta)}{2}\left\{-3(-k \eta)^{-1} \sin (-k \eta)+3 \cos (-k \eta)+(-k \eta) \sin (-k \eta)\right\} \\
& \left.+\frac{\beta(-k \eta)}{2}\left\{-3(-k \eta)^{-1} \cos (-k \eta)-3 \sin (-k \eta)+(-k \eta) \cos (-k \eta)\right\}\right],
\end{aligned}
$$

for $H r_{c} \neq 1$ and

$$
\begin{aligned}
\mathcal{R}_{(b)\left(m^{2}=2 H^{2}\right)=} & \frac{C_{1} H^{2}}{12 k}\left[-6(-k \eta)^{-1} \sin (-k \eta)\right. \\
& +\tilde{\alpha}(-k \eta)\left\{-3(-k \eta)^{-1} \sin (-k \eta)+3 \cos (-k \eta)+(-k \eta) \sin (-k \eta)\right\} \\
& \left.+\tilde{\beta}(-k \eta)\left\{-3(-k \eta)^{-1} \cos (-k \eta)-3 \sin (-k \eta)+(-k \eta) \cos (-k \eta)\right\}\right]
\end{aligned}
$$

for $H r_{c}=1$.

We should note that there are homogeneous solutions that satisfy $\mathcal{G}=0$. These homogeneous solutions also induce curvature perturbations on the brane.

\section{VAN DAM-VELTMAN-ZAKHAROV DISCONTINUITY}

\section{A. Curvature perturbation on the brane in high energy limit}

Since the background cosmology given by Eq. (3) recovers the conventional four-dimensional cosmology based on the general relativity at high energies, we expect that the gravity behaves like four-dimensional general relativity in 
this limit. Here, we show this fact analytically using our solutions. If we take the limit $H r_{c} \rightarrow \infty$ for fixed $-k \eta$, by using the formula Eq. (34), the sum of the infinite ladder of the discrete modes can be performed and we obtain

$$
\mathcal{R}_{(b)}=-A_{(b)}=-\frac{H}{4 k r_{c}}\left(C_{1} \cos (-k \eta)+C_{2} \sin (-k \eta)\right),
$$

in both branches, which agree with the solutions in general relativity Eqs. (B18), (B19). Therefore, we see that four-dimensional general relativity is recovered in this high energy limit. This confirms the result first obtained in Ref. [24].

In this limit, the brane bending vanishes $\xi=-r_{c}\left(A_{(b)}+\mathcal{R}_{(b)}\right)=0$. We can understand this result in a very simple way as follows. In terms of $A$ and $\mathcal{R}, A_{(b)}$ and $\mathcal{R}_{(b)}$ are given by

$$
A_{(b)}+\mathcal{R}_{(b)}=\frac{1}{1-2 \epsilon H r_{c}}(A+\mathcal{R}) .
$$

Then at high energies $H r_{c} \gg 1$, the brane bending mode is shielded by the curvature of the brane [18].

However, this argument holds only for $H r_{c} \rightarrow \infty$. For a finite $H r_{c}$, if we do not fix $-k \eta$ and take large enough $-k \eta$, we can no longer perform the summation of the infinite ladder of the mode and obtain the solutions (53). Thus the theory deviates from general relativity. This is related with the well-known van Dam-Veltman-Zakharov (vDVZ) discontinuity 29] first discussed in the context of the Pauli-Fierz theory for a massive spin two field [30] and also in DGP model []].

\section{B. van Dam-Veltman-Zakharov discontinuity}

Let us consider small scales limit $-k \eta \gg 1$. In this region, $(0,0)$ component of the junction conditions Eqs. (14) and the solution for the brane bending give

$$
\begin{aligned}
& \frac{2}{\kappa_{4}^{2}} \frac{k^{2}}{a^{2}} \mathcal{R}_{(b)}=\dot{\phi} \delta \dot{\phi}-\frac{2}{\kappa^{2}} \frac{k^{2}}{a^{2}} \xi+\frac{k^{2}}{a^{3}} \Omega^{\prime}, \\
& A_{(b)}+\mathcal{R}_{(b)}=-\frac{1}{r_{c}} \xi .
\end{aligned}
$$

On small scales, we can assume $H \Omega^{\prime} \ll\left(k^{2} / a^{2}\right) \Omega$, Then, Eq. (55) and (56) becomes completely the same as the one obtained from Brans-Dicke (BD) theory (B12) and (B15) [19], provided that

$$
\delta \varphi=\left(1 / \kappa^{2}\right) \xi .
$$

Thus in general we do not recover four-dimensional general relativity but BD theory. This is due to the fact that four-dimensional gravity is recovered by a continuum of massive states of five-dimensional graviton.

In the high-energy limit, we have essentially shown that the BD parameter becomes infinity for $H r_{c} \rightarrow \infty$. This results remind us that in the case of massive gravity, if we introduce a cosmological constant $\Lambda_{(4)}$, the vDVZ discontinuity disappears if the limits $m \rightarrow 0$ is taken for fixed $\Lambda_{(4)}[31,32,33$.

For a finite $H r_{c}$, the theory is not described exactly by general relativity and this difference shows up at $-k \eta \rightarrow \infty$. Then we need to know what kind of BD theory is realized for a fixed $H r_{c}$. The value of the BD parameter $\omega$ is obtained only by solving the equation of the motion for the BD scalar field (B11) because it is the only equation which includes the $\mathrm{BD}$ parameter. In the $\mathrm{BD}$ theory, the $\mathrm{BD}$ scalar obeys a simple four-dimensional equation of motion. However, in the brane-world model, the behavior of the brane bending mode can not be obtained unless we solve the five-dimensional perturbations. Now, since we have a solution for $\Omega$, we can find the solutions for the brane bending mode. In the next section, we identify the BD parameter using the solution for the brane bending.

\section{COMPARISON WITH BRANS-DICKE THEORY}

In this section, we derive the solutions for the brane bending mode and identify the BD parameter for the effective theory on small scales. 


\section{A. Brane bending mode}

Here, we show the solutions for the brane bending mode $\xi$ in - branch and + branch separately.

- branch $(\epsilon=-1)$

In - branch $(\epsilon=-1)$, the brane bending $\xi$ is given as

$$
\begin{aligned}
\xi= & -\frac{\sqrt{2 \pi}}{3} \frac{C_{1} H}{k} \frac{H r_{c}}{\left(2 H r_{c}+1\right)} \sum_{\ell=0}^{\infty} \frac{(-1)^{\ell}\left(2 \ell+\frac{1}{2}\right)}{(2 \ell+1)\left\{2(\ell+1) H r_{c}+1\right\}} \\
& \times\left[3(\ell+1)(2 \ell+1)(-k \eta)^{-\frac{1}{2}} J_{2 \ell+\frac{1}{2}}(-k \eta)-2(2 \ell+1)(-k \eta)^{\frac{1}{2}} J_{2 \ell-\frac{1}{2}}(-k \eta)+(-k \eta)^{\frac{3}{2}} J_{2 \ell-\frac{3}{2}}(-k \eta)\right] \\
& -\frac{\sqrt{2 \pi}}{3} \frac{C_{2} H}{k} \frac{H r_{c}}{\left(2 H r_{c}+1\right)} \sum_{\ell=0}^{\infty} \frac{(-1)^{\ell}\left(2 \ell+\frac{3}{2}\right)}{2(\ell+1)\left\{(2 \ell+3) H r_{c}+1\right\}} \\
& \times\left[3(\ell+1)(2 \ell+3)(-k \eta)^{-\frac{1}{2}} J_{2 \ell+\frac{3}{2}}(-k \eta)-2(2 \ell+2)(-k \eta)^{\frac{1}{2}} J_{2 \ell+\frac{1}{2}}(-k \eta)+(-k \eta)^{\frac{3}{2}} J_{2 \ell-\frac{1}{2}}(-k \eta)\right]
\end{aligned}
$$

$+\operatorname{branch}(\epsilon=1)$

In + branch $(\epsilon=1)$, the brane bending $\xi$ is given as

$$
\begin{aligned}
\xi= & \xi_{\left(m^{2}=2 H^{2}\right)} \\
& -\frac{\sqrt{2 \pi}}{3} \frac{C_{1} H}{k} \frac{H}{2 H r_{c}-1} \sum_{\ell=1}^{\infty} \frac{(-1)^{\ell}\left(2 \ell+\frac{1}{2}\right)}{2 \ell\left\{(2 \ell-1) H r_{c}+1\right\}} \\
& \times\left[-3(\ell+1)(2 \ell+1)(-k \eta)^{-\frac{1}{2}} J_{2 \ell+\frac{1}{2}}+2(2 \ell+1)(-k \eta)^{\frac{1}{2}} J_{2 \ell-\frac{1}{2}}-(-k \eta)^{\frac{3}{2}} J_{2 \ell-\frac{3}{2}}\right] \\
& -\frac{\sqrt{2 \pi}}{3} \frac{C_{2} H}{k} \frac{H r_{c}}{2 H r_{c}-1} \sum_{\ell=0}^{\infty} \frac{(-1)^{\ell}\left(2 \ell+\frac{3}{2}\right)}{(2 \ell+1)\left\{2 \ell H r_{c}+1\right\}} \\
& \times\left[-3(\ell+1)(2 \ell+3)(-k \eta)^{-\frac{1}{2}} J_{2 \ell+\frac{3}{2}}+2(2 \ell+2)(-k \eta)^{\frac{1}{2}} J_{2 \ell+\frac{1}{2}}-(-k \eta)^{\frac{3}{2}} J_{2 \ell-\frac{1}{2}}\right],
\end{aligned}
$$

where for $H r_{c} \neq 1$

$$
\begin{aligned}
\xi_{\left(m^{2}=2 H^{2}\right)=} & -\frac{C_{1} H}{6 k} \frac{H r_{c}}{\left(2 H r_{c}-1\right)\left(H r_{c}-1\right)}\left[3(-k \eta)^{-1} \sin (-k \eta)\right. \\
& +\alpha(-k \eta)\left\{3(-k \eta)^{-1} \sin (-k \eta)-3 \cos (-k \eta)-(-k \eta) \sin (-k \eta)\right\} \\
& \left.+\beta(-k \eta)\left\{3(-k \eta)^{-1} \cos (-k \eta)+3 \sin (-k \eta)-(-k \eta) \cos (-k \eta)\right\}\right],
\end{aligned}
$$

and for $H r_{c}=1$

$$
\begin{aligned}
\xi_{\left(m^{2}=2 H^{2}\right)=} & \frac{C_{1} H}{6 k}\left[\tilde{\alpha}(-k \eta)\left\{3(-k \eta)^{-1} \sin (-k \eta)-3 \cos (-k \eta)-(-k \eta) \sin (-k \eta)\right\}\right. \\
& +\tilde{\beta}(-k \eta)\left\{3(-k \eta)^{-1} \cos (-k \eta)+3 \sin (-k \eta)-(-k \eta) \cos (-k \eta)\right\} \\
& +\frac{3}{2} \alpha(-k \eta)(-k \eta)^{-1} \sin (-k \eta)+\frac{3}{2} \beta(-k \eta)(-k \eta)^{-1} \cos (-k \eta) \\
& \left.+3(-k \eta)^{-1} \sin (-k \eta)\right]
\end{aligned}
$$

There are homogeneous solutions that satisfy $\mathcal{G}=0$. These are given by

$$
\Omega \propto(-k \eta)^{-3 / 2} Z_{\nu}(-k \eta)
$$

where $Z_{\nu}$ is a linear combination of Bessel functions and $\nu$ is determined by the spectrum of massive modes. On small scales, regardless of the value of $\nu$, the solution for the brane bending from the homogeneous solutions are given by

$$
\xi=d_{1}(-k \eta) \sin (-k \eta)+d_{2}(-k \eta) \cos (-k \eta)
$$

where $d_{1}$ and $d_{2}$ are arbitrary constants. 


\section{B. Perturbation of Brans-Dicke scalar}

Now we examine the behavior of $\delta \varphi$ in the BD theory in the presence of the scalar field $\phi$, whose dynamics is completely the same as the brane-world model. Since we are interested in the behavior of $\delta \varphi$ on sub-horizon scale, we take the small scale limit. Then, from Eqs. (30) and (33), $\phi^{\prime} \delta \phi^{\prime}$ can be evaluated as

$$
\phi^{\prime} \delta \phi^{\prime}=-\frac{k H}{2 r_{c} \kappa_{4}^{2}}\left[C_{1} \cos (-k \eta)+C_{2} \sin (-k \eta)\right],
$$

where in order to obtain Eq. [64), we used the fact that $(\ddot{\phi} / \dot{\phi}) \ll H \ll k / a$ in the de Sitter background and subhorizon scale.

This term serves as a source term in the equation of motion of the perturbed Brans-Dicke scalar field B11 which can be simplified in the de Sitter background as

$$
\delta \varphi^{\prime \prime}+\frac{\eta}{2} \delta \varphi^{\prime}-\nabla^{2} \delta \varphi=-2 \zeta^{2} \phi^{\prime} \delta \phi^{\prime}
$$

where $\zeta$ is a constant related with the BD parameter $\omega$ as $\zeta^{2}=(6+4 \omega)^{-1}$. Then by solving Eq. (65) and keeping leading terms, we obtain

$$
\begin{aligned}
\delta \varphi= & \tilde{d}_{1}(-k \eta) \sin (-k \eta)+\tilde{d}_{2}(-k \eta) \cos (-k \eta) \\
& +\frac{1}{2}\left[-\bar{C}_{2}(-k \eta) \sin (-k \eta)+\bar{C}_{1}(-k \eta) \cos (-k \eta)\right] \operatorname{Si}(-2 k \eta) \\
& +\frac{1}{2}\left[-\bar{C}_{1}(-k \eta) \sin (-k \eta)-\bar{C}_{2}(-k \eta) \cos (-k \eta)\right] C i(-2 k \eta) \\
& +\frac{1}{2}\left[\bar{C}_{1}(-k \eta) \ln (-k \eta) \sin (-k \eta)-\bar{C}_{2}(-k \eta) \ln (-k \eta) \cos (-k \eta)\right],
\end{aligned}
$$

where $\tilde{d}_{1}$ and $\tilde{d}_{2}$ are integration constants related with the homogeneous solutions and $\bar{C}_{1}$ and $\bar{C}_{2}$ are given by

$$
\bar{C}_{1}=\frac{C_{1} H \zeta^{2}}{r_{c} \kappa_{4}^{2} k}, \quad \bar{C}_{2}=\frac{C_{2} H \zeta^{2}}{r_{c} \kappa_{4}^{2} k} .
$$

Note that the homogeneous solutions behave in the same ways as the brane bending mode coming from homogeneous solutions for $\Omega$ (63). These homogeneous solutions are fixed by appropriate boundary conditions.

\section{Numerical results}

Here, we identify the value of the Brans-Dicke parameter $\omega$. For this purpose, we evaluate Eqs. (58), (59) and (66) numerically. In practice, we must approximate the infinite sum to proceed the calculations. In our previous paper [5], we checked that we can approximate the infinite summation by introducing a cut-off $\ell_{c}$ into the summation because Bessel function satisfies $J_{\nu}(z) \rightarrow 0$ for $\nu>z$. Therefore, as long as we start from a finite time $-k \eta_{i}$, we can approximate the infinite ladder of the modes by introducing sufficiently large cut-off $\ell_{c}$ and the result is insensitive to $\ell_{c}$.

Here, we compare the part of the numerical solutions including $C_{1}$ of Eqs. (59) with (66). The same result applies to the solution including $C_{2}$. For the numerical calculation, we construct the following dimensionless quantities;

$$
\begin{gathered}
\tilde{\xi}_{1}=\frac{k}{2 C_{1} H} \frac{1}{H r_{c}} \xi_{1}=-\frac{1}{2} \frac{k}{C_{1} H^{2}}\left(A_{(b) 1}+\mathcal{R}_{(b) 1}\right), \\
\delta \tilde{\varphi}_{1}=\frac{\kappa^{2} k}{2 C_{1} H} \delta \varphi_{1}=-\frac{1}{2} \frac{k}{C_{1} H^{2}}\left(A_{\mathrm{BD} 1}+\mathcal{R}_{\mathrm{BD} 1}\right),
\end{gathered}
$$

where the subscript 1 denotes that they correspond to the part including $C_{1}$.

Figure 3 shows $\tilde{\xi}_{1}$ (solid line) and $\delta \tilde{\varphi}_{1}$ (dotted line) as functions of $-k \eta$ in the scale smaller than the Hubble scale $(-k \eta=1)$. The Brans-Dicke parameter is chosen as $3 H r_{c}$. Here we take $H r_{c}=2$ and choose the amplitudes of the homogeneous solutions for $\delta \tilde{\varphi}_{1}, \tilde{d}_{1}$ and $\tilde{d}_{2}$ so that it agrees to $\tilde{\xi}_{1}$ with $d_{1}=d_{2}=0$ at $-k \eta=200$. We see that the behavior of perturbations on scales much smaller than the Hubble scale is well described by the Brans-Dicke theory with $\omega=3 H r_{c}$. It can also be seen that, at least for $-k \eta<120$, the deviation of $\tilde{\xi}_{1}$ from $\delta \tilde{\varphi}_{1}$ become large and the 


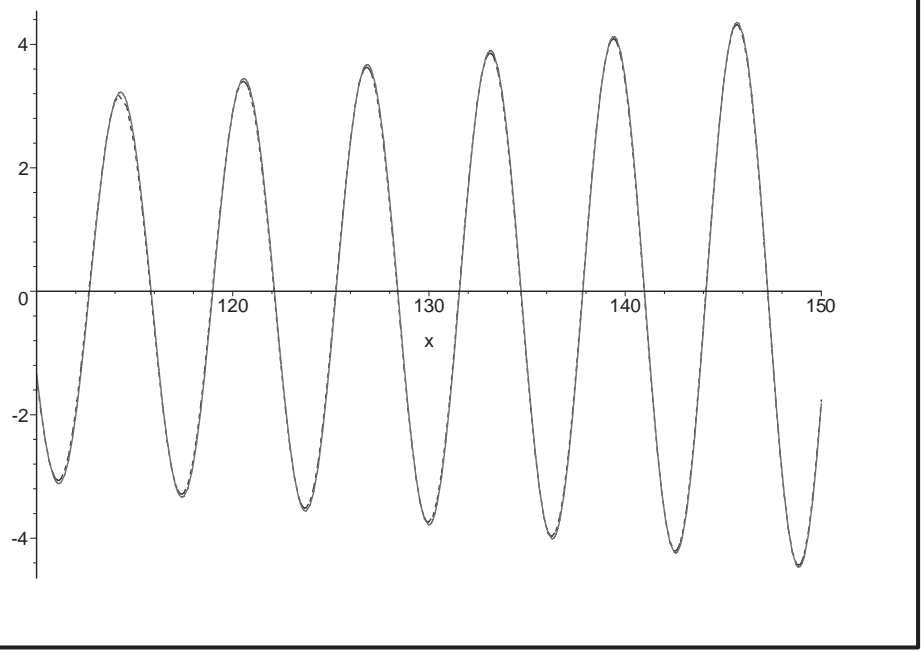

FIG. 3: $\tilde{\xi}_{1}$ (solid line) and $\delta \tilde{\varphi}_{1}$ (dotted line) as functions of $x=-k \eta$. The Brans-Dicke parameter is $3 H r_{c}$. Here we take $H r_{c}=2$. The dotted line completely coincides with the solid line on small scales (large $x$ ). The deviation become obvious around $x<120$.

solution is no longer well described by the Brans-Dicke theory with $\omega=3 H r_{c}$. Figure 4 also shows $\tilde{\xi}_{1}$ (solid line) and $\delta \tilde{\varphi}_{1}$ (dotted line) with $\omega=3 H r_{c}$ as functions of $-k \eta$ for $H r_{c}=0.1$. We also choose the homogeneous solutions so that both coincide at $-k \eta=200$ in the same way. Again we see the solution is well described by the BD theory with $\omega=3 H r_{c}$, but the deviation of $\tilde{\xi}_{1} \delta$ from $\tilde{\varphi}_{1}$ become large for $-k \eta<180$. This is natural because, for $H r_{c}=0.1, r_{c}$ becomes smaller than the horizon scale (corresponding to $-k \eta=10$ ).

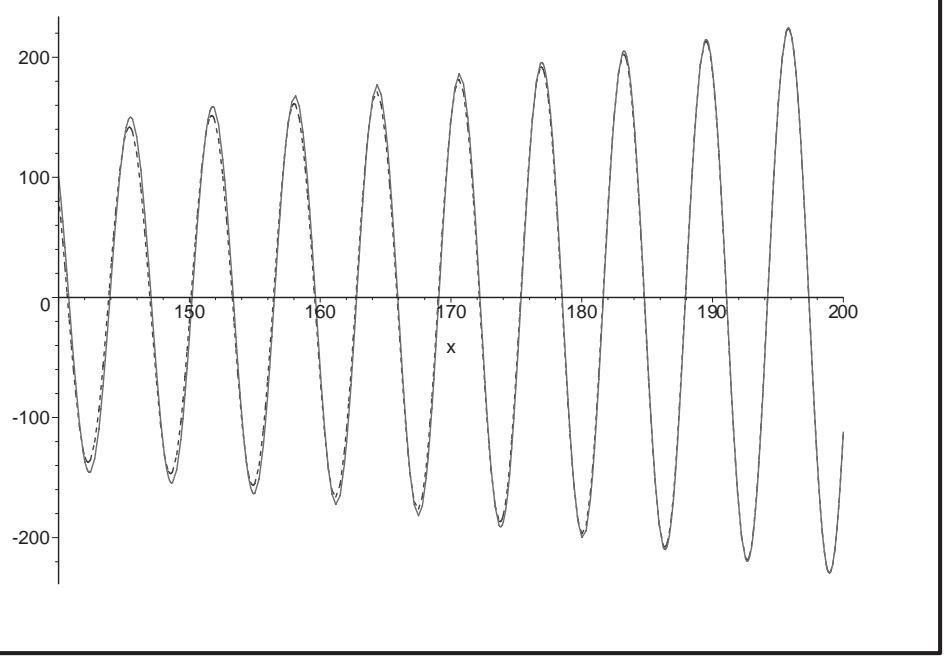

FIG. 4: $\tilde{\xi}_{1}$ (solid line) and $\delta \tilde{\varphi}_{1}$ (dotted line) as functions of $x=-k \eta$. The Brans-Dicke parameter is $3 H r_{c}$. Here we take $H r_{c}=0.1$. The dotted line completely coincides with the solid line on small scales (large $x$ ). The deviation become obvious around $x \sim 180$.

These results are consistent with Ref. 18, 19]. In Ref. 18], it is shown that the linear growth of density contrast in non-relativistic spherical collapse of dust is described the Brans-Dicke theory with $\omega=3 H r_{c}$ for $1 / H \gg r$ when $H r_{c}$ is greater than 1.

Even though we do not show the results explicitly here, we can confirm that, in the + branch, similar results can be obtained that the perturbations can be described by the Brans-Dicke theory with $\omega=-3 H r_{c}$ on small scale. This is also consistent with [18, 19]. In the Brans-Dicke theory, the Brans-Dicke scalar $\varphi$ becomes a ghost if the Brans-Dicke 
parameter satisfies $\omega+3 / 2<0$. This result implies that the brane bending $\xi$ is a ghost for $H r_{c}>1$ in + branch.

\section{CURVATURE PERTURBATION ON THE BRANE ON LARGE SCALES}

\section{A. Solutions on Large scales}

In this section, we study the behavior of perturbations on large scales $(-k \eta) \ll 1$. In this limit, the solution for $\Omega$ is dominated by the $m^{2}=2 H^{2}$ mode. From Eq. (30) in the limit $(-k \eta) \ll 1, C_{1}$ is related with the scalar field perturbation as

$$
\kappa^{2} \delta \phi=\frac{C_{1}}{k} \frac{H^{2}}{\dot{\phi}}
$$

in both branches.

In the following, we express the curvature perturbation on the brane as the perturbation of the scalar field in this limit in both branches.

- branch $(\epsilon=-1)$

In this limit, the $m^{2}=2 H^{2}$ mode of $\mathcal{R}_{(b)\left(m^{2}=2 H^{2}\right)}$ is,

$$
\mathcal{R}_{(b)\left(m^{2}=2 H^{2}\right)}=-\frac{C_{1}}{2 k} \frac{H^{2}}{\left(2 r_{c} H+1\right)},
$$

where we have used the fact that $J_{1 / 2}(-k \eta) \rightarrow(-k \eta)^{1 / 2} \sqrt{2 / \pi}$ as $(-k \eta) \rightarrow 0$. Then we can relate the perturbation of the scalar field and the curvature perturbation as

$$
\mathcal{R}_{(b)}=-\kappa_{4,-\mathrm{eff}}^{2} \frac{\dot{\phi}}{2 H} \delta \phi, \quad \kappa_{4,-\mathrm{eff}}^{2}=\kappa_{4}^{2} \frac{2 H r_{c}}{\left(2 H r_{c}+1\right)},
$$

which is the same as the standard four-dimensional result (see Eq. (B21)), except for the overall normalization of the effective gravitational constant. If we take the limit $r_{c} H \rightarrow \infty, \kappa_{4},-$ eff coincides with $\kappa_{4}$ and we recover fourdimensional results. We can also show that $\mathcal{R}_{(b)}=-A_{(b)}$.

$+\operatorname{branch}(\epsilon=1)$

In this limit, for $H r_{c} \neq 1$, the $m^{2}=2 H^{2}$ mode of $\mathcal{R}_{(b)\left(m^{2}=2 H^{2}\right)}$ becomes

$$
\mathcal{R}_{(b)\left(m^{2}=2 H^{2}\right)}=-\frac{C_{1}}{2 k} \frac{H^{2}}{\left(2 H r_{c}-1\right)} \frac{H r_{c}}{\left(H r_{c}-1\right)},
$$

where we have used the fact that

$$
\begin{aligned}
\alpha(-k \eta) & \rightarrow 2 \ln (-k \eta), \\
\beta(-k \eta) & \rightarrow 2 k \eta \text { as }-k \eta \rightarrow 0 .
\end{aligned}
$$

Strictly speaking, the asymptotic value of $\alpha(-k \eta)$ and $\beta(-k \eta)$ contain an arbitrary integration constants (see Eqs. (A7) and (A8). It was shown in Ref. 34, 39] that this degree of freedom corresponds to a radion and dark radiation. In the following, we neglect this contribution.

Then, for $H r_{c} \neq 1$, we can relate the perturbation of the scalar field and the curvature perturbation as

$$
\mathcal{R}_{(b)}=-\kappa_{4,+\mathrm{eff}}^{2} \frac{H r_{c}}{\left(H r_{c}-1\right)} \frac{\dot{\phi}}{2 H} \delta \phi, \quad \kappa_{4,+\mathrm{eff}}^{2}=\kappa_{4}^{2} \frac{2 H r_{c}}{\left(2 H r_{c}-1\right)}
$$

On the other hand we get

$$
A_{(b)}=\kappa_{4,+\mathrm{eff}}^{2} \frac{\dot{\phi}}{2 H} \delta \phi
$$

Again, if we take the limit $H r_{c} \rightarrow \infty$, we recover the four-dimensional results. 


\section{B. Self-accelerating background}

Similarly, for $H r_{c}=1, \mathcal{R}_{(b)\left(m^{2}=2 H^{2}\right)}$ becomes

$$
\mathcal{R}_{(b)\left(m^{2}=2 H^{2}\right)}=\frac{C_{1} H^{2}}{2 k}(-2+\ln (-k \eta)),
$$

where we have used the fact that

$$
\begin{aligned}
& \tilde{\alpha}(-k \eta) \rightarrow(\ln (-k \eta))^{2}, \\
& \tilde{\beta}(-k \eta) \rightarrow-2 k \eta-2 \ln (-k \eta)(-k \eta) \text { as } \quad-k \eta \rightarrow 0,
\end{aligned}
$$

as well as Eqs. (73) and (74). In terms of the perturbation of the scalar field, this is given by

$$
\mathcal{R}_{(b)}=\kappa_{4}^{2} \frac{\dot{\phi}}{H}(-2+\ln (-k \eta)) \delta \phi .
$$

We also get

$$
A_{(b)}=0 .
$$

The solution for $\mathcal{R}_{(b)}$ is diverging on large scales limit $(-k \eta) \rightarrow 0$. In the next section, we study whether this means the physical instability on the brane or not.

\section{Effective theory on a brane}

In order to see the origin of the difference between + branch and - branch, it is useful to consider effective covariant gravitational field equations on the brane derived by projecting the five-dimensional Einstein equations and applying the Israel junction conditions with reflection symmetry at the brane [36, 37],

$$
G_{\mu \nu}=\left(16 \pi G r_{c}\right)^{2} \Pi_{\mu \nu}-E_{\mu \nu}
$$

where

$$
\begin{aligned}
\tilde{T}_{\mu \nu} & =T_{\mu \nu}-(8 \pi G)^{-1} G_{\mu \nu}, \\
\Pi_{\mu \nu} & =-\frac{1}{4} \tilde{T}_{\mu \alpha} \tilde{T}_{\nu}{ }^{\alpha}+\frac{1}{12} \tilde{T}_{\alpha}{ }^{\alpha} \tilde{T}_{\mu \nu}+\frac{1}{24}\left[3 \tilde{T}_{\alpha \beta} \tilde{T}^{\alpha \beta}-\left(\tilde{T}_{\alpha}{ }^{\alpha}\right)^{2}\right] g_{\mu \nu},
\end{aligned}
$$

and $E_{\mu \nu}$ is the trace-free projection of the five-dimensional Weyl tensor. In the background, we take $E_{\mu \nu}=0$ by assuming a Minkowski bulk. However, perturbations of $E_{\mu \nu}$ are not necessarily vanishing. For perturbations, the effective equations are

$$
\begin{gathered}
\left(1-\frac{1}{2 H r_{c}}\right)\left\{-6 H\left(\dot{\mathcal{R}}_{(b)}-H A_{(b)}\right)-\frac{2 k^{2}}{a^{2}} \mathcal{R}_{(b)}\right\}=-\kappa_{4}^{2} \delta \rho+\frac{\kappa_{4}^{2}}{2 H r_{c}} \delta \rho_{E}, \\
2\left(1-\frac{1}{2 H r_{c}}\right)\left(\dot{\mathcal{R}}_{(b)}-H A_{(b)}\right)=\kappa_{4}^{2} a \delta q-\frac{\kappa_{4}^{2}}{2 H r_{c}} a \delta q_{E}, \\
-\frac{1}{a^{2}}\left(1-\frac{1}{2 H r_{c}}\right)\left(A_{(b)}+\mathcal{R}_{(b)}\right)=\kappa_{4}^{2} \delta \pi-\frac{\kappa_{4}^{2} \delta \pi_{E}}{2 H r_{c}} .
\end{gathered}
$$

Substituting the solution for $\mathcal{R}_{(b)}$ and $A_{(b)}$, we find the perturbations of Weyl tensor vanish in - branch. In + branch, $\delta \rho_{E}=\delta q_{E}=0$, but there is a non-trivial anisotropic stress;

$$
\begin{aligned}
\delta \pi_{E} & =-\frac{1}{a^{2}} \frac{r_{c} \dot{\phi}}{\left(H r_{c}-1\right)} \delta \phi, \quad H r_{c} \neq 1, \\
\delta \pi_{E} & =\frac{1}{a^{2}} \frac{\dot{\phi}}{H}(\log (-k \eta)-2) \delta \phi, \quad H r_{c}=1 .
\end{aligned}
$$


Since $a \propto(-k \eta)^{-1}$, even though $\ln (-k \eta)$ diverges as $-k \eta \rightarrow 0$, the anisotropic stress from perturbations of $E_{\mu \nu}$ does not diverge in the limit $-k \eta \rightarrow 0$.

Since gauge invariant perturbations of Weyl tensor do not diverge, the divergence of the curvature perturbation in longitudinal gauge Eq. (80) does not imply the instability of the spacetime itself and the divergence is caused simply by a bad choice of the gauge.

In order to show this, let us consider a four-dimensional gauge transformation,

$$
\eta \rightarrow \eta-\epsilon^{\eta}, \quad x^{i} \rightarrow x^{i}-\epsilon^{, i} .
$$

By choosing $\partial_{\eta} \epsilon=\epsilon^{\eta}$, the metric perturbations are transformed as

$$
d s^{2}=a^{2}\left\{-\left(1+2 \tilde{A}_{(b)}\right) d \eta^{2}+\left[\left(1+2 \tilde{\mathcal{R}}_{(b)}\right) \delta_{i j}+\tilde{E}_{(b), i j}\right] d x^{i} d x^{j}\right\},
$$

where

$$
\begin{aligned}
& \tilde{A}_{(b)}=A_{(b)}-\partial_{\eta} \epsilon^{\eta}-\left(\partial_{\eta} \ln a\right) \epsilon^{\eta}, \\
& \tilde{\mathcal{R}}_{(b)}=\mathcal{R}_{(b)}-\left(\partial_{\eta} \ln a\right) \epsilon^{\eta}, \\
& \tilde{E}_{(b)}=-\epsilon .
\end{aligned}
$$

For $H r_{c} \neq 1$, we can eliminate the singular part in $\mathcal{R}_{(b)}$ in the limit $H r_{c} \rightarrow 1$ by choosing

$$
\epsilon^{\eta}=-\kappa_{4,+\mathrm{eff}}^{2} \frac{1}{\left(H r_{c}-1\right)} \frac{\dot{\phi}}{2 k H}(-k \eta) \delta \phi
$$

Then the resultant metric perturbations are given by

$$
\begin{aligned}
\tilde{\mathcal{R}}_{(b)} & =-\tilde{A}_{(b)}=-\kappa_{4,+\mathrm{eff}}^{2} \frac{\dot{\phi}}{2 H} \delta \phi, \\
\partial_{\eta} \tilde{E}_{(b)} & =\kappa_{4,+\mathrm{eff}}^{2}(-k \eta) \frac{1}{\left(H r_{c}-1\right)} \frac{\dot{\phi}}{2 k H} \delta \phi .
\end{aligned}
$$

For $H r_{c}=1$, we can also eliminate the growing part of $\mathcal{R}_{(b)}$ by choosing

$$
\epsilon^{\eta}=\frac{\kappa_{4}^{2} \dot{\phi}}{k H} \delta \phi(-k \eta)(\ln (-k \eta)-1) .
$$

Then, the resultant metric perturbations become

$$
\begin{aligned}
\tilde{\mathcal{R}}_{(b)} & =-\tilde{A}_{(b)}=-\kappa_{4}^{2} \frac{\dot{\phi}}{H} \delta \phi \\
\partial_{\eta} \tilde{E}_{(b)} & =-\frac{\kappa_{4}^{2} \dot{\phi}}{k H}(-k \eta)(\ln (-k \eta)-1) \delta \phi .
\end{aligned}
$$

At late times $-k \eta \rightarrow 0, E_{(b)}$ behaves as

$$
\tilde{E}_{(b)} \propto(-k \eta)^{2} \ln (-k \eta) \rightarrow 0 .
$$

Thus we can find a gauge where all perturbations remain small and so there is no gravitational instability.

\section{CONCLUSION}

In this paper, we studied inflaton perturbations confined to a de Sitter brane with induced gravity in a fivedimensional Minkowski spacetime.

For a vacuum brane, the spin- 0 mode appears as a discrete bulk mode with $m^{2}=2 H^{2}$ in the + branch, while in the - branch there are no normalizable solutions for the spin-0 modes. Since there is another discrete bulk mode (helicity-0 mode of spin-2 perturbation) with mass $m^{2}=H^{2}\left(3 H r_{c}-1\right)\left(H r_{c}\right)^{-2}$ in the + branch, there is a resonance between the spin- 0 mode and the helicity- 0 mode of spin- 2 perturbation for $H r_{c}=1$. In this paper we introduced inflaton perturbations on a brane. Then an infinite ladder of discrete modes with $m^{2}=-2(2 \ell-1)(\ell+1) H^{2}$ and 
$m^{2}=-2 \ell(2 \ell+3) H^{2}$ are excited. Since there is a mode with $m^{2}=2 H^{2}$ regardless of the value of $H r_{c}$, in the + branch the resonance inevitably appears. We obtained the solutions for the curvature perturbation on the brane $\mathcal{R}_{(b)}$ and studied their behavior.

At high energies, $H r_{c} \rightarrow \infty$, we have confirmed that, in both branches, the four-dimensional general relativity solutions are recovered from the infinite sum of the modes. This results remind us that in the case of massive gravity, if we introduce a cosmological constant $\Lambda_{(4)}$, the vDVZ discontinuity disappears if the limits $m \rightarrow 0$ is taken for fixed $\Lambda_{(4)}$ 31, 32, 33]. However, this argument holds only for $H r_{c} \rightarrow \infty$. For a finite $H r_{c}$, if we do not fix $-k \eta$ and take large enough $-k \eta$, we can no longer perform the summation of the infinite ladder of the modes and the theory deviate from four-dimensional general relativity. In fact, on small scales, for a fixed $H r_{c}$ we can see that the junction condition becomes the same as the gravitational field equations in Brans-Dicke theory where the brane bending mode acts as the BD scalar.

Then, in order to identify the BD parameter for the effective BD theory, we compared the solutions for Brans-Dicke scalar field and the brane bending mode. We have confirmed that, on sufficiently small scales, gravity is well described by the Brans-Dicke theory with $\omega=-3 H r_{c}$ in + branch and $\omega=3 H r_{c}$ in - branch. This is consistent with the previous work [18, 19] and the existence of the ghost in + branch is confirmed.

We also studied the large scales perturbations. In - branch, the solutions agree with four-dimensional GR with a modified gravitational constant. In + branch, there is an additional contribution to $\mathcal{R}_{(b)}$ due to the resonance, which diverges on large scales for $H r_{c}=1$. We identified this contribution as the effect of the Weyl anisotropic stress and showed that the anisotropic stress itself does not diverge. In fact, we can find a suitable gauge where all metric perturbations remain small, so the resonance does not lead to a gravitational instability on the brane.

We make comments on future applications of our results. In - branch, if $r_{c} \ll H^{-1}$, small scale perturbations $a / k \ll r_{c}$ can be described by the four-dimensional Brans-Dicke theory. If the perturbations approaches to $r_{c}$, the gravity becomes five-dimensional and we expect significant effects from the coupling to five-dimensional metric perturbations [5]. Unlike the Randall-Sundrum model where the small scales perturbations are always coupled to five-dimensional perturbations and a quantum vacuum state is hard to be specified [5, 38, 39], we can specify a vacuum state without ambiguity based on the four-dimensional BD theory on sufficiently small scales. Then we can estimate the effect of the coupling to five-dimensional gravity without ambiguity.

Finally we should emphasize that our analysis is limited to linear perturbations. It has been shown that we need to take into account the non-linearity of the brane bending modes before the gravity becomes non-linear. Actually, we can recover four-dimensional general relativity due to this non-linearity [26, 40, 41, 42, 43, 44, 45]. Ref. [18] suggested that this non-linear scale becomes very small at high energies by the factor $1-\epsilon 2 H r_{c}$, which is the same factor seen in Eq. (54). The non-linear interactions of the brane bending mode in de Sitter spacetime deserve further study.

\section{Acknowledgments}

SM is grateful to the ICG, Portsmouth for their hospitality when this work was initiated. SM is supported in part by the Japan Society for Promotion of Science (JSPS) Research Fellowship. KK is supported by PPARC.

\section{APPENDIX A: DERIVATION OF $\Omega_{m^{2}=2 H^{2}}$ IN + BRANCH}

In order to obtain the mode corresponding to $m^{2}=2 H^{2}$, we must prepare the solution other than $N(y)$, since the junction conditions cannot be satisfied by this solution. We find that depending on whether $H r_{c}=1$ or not, there are two types of the solutions.

\section{$H r_{c} \neq 1$}

First, we consider the case $H r_{c} \neq 1$. In this case, we assume the following form of the solution:

$$
\Omega_{\left(m^{2}=2 H^{2}\right)}=(1+H y) P(t)+(1+H y) \ln (1+H y) Q(t) .
$$

By substituting Eq. (A1) into the junction condition (33), we obtain the following relation;

$$
\left(1-2 H r_{c}\right) H Q(t)-r_{c}\left(2 H^{2}-m^{2}\right) P(t)=C_{1} \sqrt{\frac{\pi}{2}}(-k \eta)^{-\frac{3}{2}} J_{\frac{1}{2}}(-k \eta) .
$$

It is worth noting that in Eq. (A2), since $P(t)$ is no longer $(-k \eta)^{-3 / 2} J_{\frac{1}{2}}(-k \eta), m^{2}$ is not a constant but a differential operator. On the other hand, we find that the equation of the motion in the bulk is satisfied if the following conditions 
hold:

$$
\begin{aligned}
& \left(2 H^{2}-m^{2}\right) P(t)=-H^{2} Q(t), \\
& \left(2 H^{2}-m^{2}\right) Q(t)=0 .
\end{aligned}
$$

By eliminating $P(t)$ from Eqs. (A2) and (A3), we get

$$
Q(t)=-C_{1} \frac{1}{H\left(H r_{c}-1\right)}(-k \eta)^{-2} \sin (-k \eta),
$$

and by substituting Eq. A5 into Eq. A3 we obtain

$$
P(t)=C_{1} \frac{1}{2 H\left(H r_{c}-1\right)} \frac{1}{(-k \eta)^{2}}\{\alpha(-k \eta) \sin (-k \eta)+\beta(-k \eta) \cos (-k \eta)\},
$$

where

$$
\begin{aligned}
& \alpha(-k \eta)=\int^{-k \eta} d(-k \bar{\eta})(-k \bar{\eta})^{-2} \sin (-2 k \bar{\eta}), \\
& \beta(-k \eta)=\int^{-k \eta} d(-k \bar{\eta})(-k \bar{\eta})^{-2}\{\cos (-2 k \bar{\eta})-1\} .
\end{aligned}
$$

$H r_{c}=1$

Next, we consider the case $H r_{c}=1$. In this case, we assume the following form of the solution:

$$
\Omega_{\left(m^{2}=2 H^{2}\right)}=(1+H y) P(t)+(1+H y) \ln (1+H y) Q(t)+(1+H y)(\ln (1+H y))^{2} S(t) .
$$

By substituting Eq. (A9) into the junction condition (33), we can obtain the following relation:

$$
-H Q(t)-\frac{1}{H}\left(2 H^{2}-m^{2}\right) P(t)=C_{1} \sqrt{\frac{\pi}{2}}(-k \eta)^{-\frac{3}{2}} J_{\frac{1}{2}}(-k \eta) .
$$

It is worth noting that in Eq. A10, since $P(t)$ is no longer $(-k \eta)^{-3 / 2} J_{\frac{1}{2}}(-k \eta), m^{2}$ is not a constant but a differential operator. On the other hand, we find that the equation of the motion in the bulk is satisfied if the following conditions hold:

$$
\begin{aligned}
\left(2 H^{2}-m^{2}\right) P(t) & =\left(m^{2}-3 H^{2}\right) Q(t), \\
\left(2 H^{2}-m^{2}\right) Q(t) & =-2 H^{2} S(t), \\
\left(2 H^{2}-m^{2}\right) S(t) & =0 .
\end{aligned}
$$

By eliminating $P(t)$ and $Q(t)$ from Eqs. (A11), (A12) and (A13) we obtain

$$
S(t)=-\frac{C_{1}}{2 H}(-k \eta)^{-2} \sin (-k \eta) .
$$

By substituting (A14) into (A12), we obtain

$$
Q(t)=\frac{C_{1}}{2 H} \frac{1}{(-k \eta)^{2}}\{\alpha(-k \eta) \sin (-k \eta)+\beta(-k \eta) \cos (-k \eta)\},
$$

where $\alpha(-k \eta)$ and $\beta(-k \eta)$ are defined as Eqs. (A7) and (A8). Furthermore, by substituting (A14) and (A15) into (A11), we get

$$
P(t)=-\frac{C_{1}}{2 H} \frac{1}{(-k \eta)^{2}}\{\tilde{\alpha}(-k \eta) \sin (-k \eta)+\tilde{\beta}(-k \eta) \cos (-k \eta)\}
$$

where

$$
\begin{aligned}
& \tilde{\alpha}(-k \eta)=\alpha(-k \eta)+\frac{1}{2} \int^{-k \eta} d(-k \bar{\eta})(-k \bar{\eta})^{-2}[\alpha(-k \bar{\eta}) \sin (-2 k \bar{\eta})+\beta(-k \bar{\eta})\{\cos (-2 k \bar{\eta})+1\}] \\
& \tilde{\beta}(-k \eta)=\beta(-k \eta)+\frac{1}{2} \int^{-k \eta} d(-k \bar{\eta})(-k \bar{\eta})^{-2}[\alpha(-k \bar{\eta})\{\cos (-2 k \bar{\eta})-1\}-\beta(-k \bar{\eta}) \sin (-2 k \bar{\eta})] .
\end{aligned}
$$




\section{APPENDIX B: METRIC PERTURBATIONS IN 4D BRANS-DICKE GRAVITY}

In this appendix, we briefly summarize the results for cosmological perturbations in four-dimensional Brans-Dicke theory (see [46] for a review of BD theory).

In this theory, we start with the following action:

$$
\mathcal{L}_{\mathrm{BD}}=\sqrt{-g}\left(\varphi R-\omega \frac{1}{\varphi} g^{\mu \nu} \partial_{\mu} \varphi \partial_{\nu} \varphi+L_{\text {matter }}\right),
$$

where $\varphi$ is a Brans-Dicke scalar field and $\omega$ is a Brans-Dicke coupling constant. From Eq. (B1) we can derive the field equations,

$$
\begin{aligned}
2 \varphi G_{\mu \nu} & =T_{\mu \nu}+T_{\mu \nu}^{\varphi}-2\left(g_{\mu \nu} \square-\nabla_{\mu} \nabla_{\nu}\right) \varphi, \\
\square \varphi & =\zeta^{2} T, \\
\nabla_{\mu} T^{\mu \nu} & =0,
\end{aligned}
$$

where $\zeta$ is a constant related with $\omega$ as

$$
\zeta^{-2}=6+4 \omega
$$

For perturbations, the metric is taken as

$$
d s^{2}=-(1+2 A) d t^{2}+a(t)^{2}(1+2 \mathcal{R}) \delta_{i j} d x^{i} d x^{j} .
$$

When we consider a canonical scalar field with a potential $V(\phi)$ as the matter, the field equations reduce to

$$
\begin{aligned}
& 6 \mathcal{H}^{2} \delta \varphi+4 \varphi_{0}\left[-3 \mathcal{H}\left(\mathcal{H} A-\mathcal{R}^{\prime}\right)-\nabla^{2} \mathcal{R}\right]=-\phi^{\prime 2} A+\phi^{\prime} \delta \phi^{\prime}+V,_{\phi} a^{2} \delta \phi-6 \mathcal{H} \delta \varphi^{\prime}+2 \nabla^{2} \delta \varphi, \\
& 4 \varphi_{0}\left(\mathcal{H} A-\mathcal{R}^{\prime}\right)=\phi^{\prime} \delta \phi+2 \delta \varphi^{\prime}-2 \mathcal{H} \delta \varphi, \\
& 2 \delta \varphi\left(2 \mathcal{H}^{\prime}+\mathcal{H}^{2}\right)-4 \varphi_{0}\left[\left(2 \mathcal{H}^{\prime}+\mathcal{H}^{2}\right) A+\mathcal{H} A^{\prime}-\mathcal{R}^{\prime \prime}-2 \mathcal{H} \mathcal{R}^{\prime}+\frac{1}{2} \nabla^{2}(A+\mathcal{R})\right] \\
& \quad=\phi^{\prime 2} A-\phi^{\prime} \delta \phi^{\prime}+V,_{\phi} a^{2} \delta \phi-2 \delta \varphi^{\prime \prime}-2 \mathcal{H} \delta \varphi^{\prime}+2 \nabla^{2} \delta \varphi, \\
& \varphi_{0}(A+\mathcal{R})=-\delta \varphi \\
& \delta \varphi^{\prime \prime}+2 \mathcal{H} \delta \varphi^{\prime}-\nabla^{2} \delta \varphi=2 \zeta^{2}\left[\phi^{\prime} A-\phi^{\prime} \delta \phi+2 V,_{\phi} a^{2} \delta \phi\right],
\end{aligned}
$$

where the prime here denotes the derivative with respect to conformal time $\eta$ and $\mathcal{H}=a^{\prime} / a$

If we require Eqs. (B7), (B8), (B9) and (B10) reproduces the results of GR in the limit of $\delta \varphi \rightarrow 0$ and $\omega \rightarrow \infty$, the value of $\varphi_{0}$ is set to be $\varphi_{0}=\left(1 / 2 \kappa_{4}^{2}\right)$. For the scale much smaller than the Hubble radius and the scalar field evolves slowly in the background, Eqs. (B7), (B8), (B9) and (B10) can be simplified as

$$
\begin{aligned}
-4 \varphi_{0} \nabla^{2} \mathcal{R} & =\phi^{\prime} \delta \phi^{\prime}+2 \nabla^{2} \delta \varphi, \\
-4 \varphi_{0} \mathcal{R}^{\prime} & =\phi^{\prime} \delta \phi+2 \delta \varphi^{\prime}, \\
4 \varphi_{0} \mathcal{R}^{\prime \prime}-2 \varphi_{0} \nabla^{2}(A+\mathcal{R}) & =-\phi^{\prime} \delta \phi^{\prime}+2 \nabla^{2} \delta \varphi, \\
\varphi_{0}(A+\mathcal{R}) & =-\delta \varphi .
\end{aligned}
$$

Then the equation for curvature perturbation is obtained as

$$
\mathcal{R}^{\prime \prime}-\nabla^{2} \mathcal{R}=\frac{1}{2 \varphi_{0}} \nabla^{2} \delta \varphi .
$$

If we take general relativity limit, we get

$$
\mathcal{R}^{\prime \prime}-\nabla^{2} \mathcal{R}=0
$$

and its Fourier modes of $\mathcal{R}$ are expressed as

$$
\mathcal{R}=\tilde{C}_{1} \cos (-k \eta)+\tilde{C}_{2} \sin (-k \eta),
$$

where $\tilde{C}_{1}$ and $\tilde{C}_{2}$ are integration constants [47]. The lapse function $A$ is obtained as

$$
A=-\mathcal{R}=-\tilde{C}_{1} \cos (-k \eta)-\tilde{C}_{2} \sin (-k \eta) \text {. }
$$


If we take a large scale limit, that is, $-k \eta \rightarrow 0$, from Eq. (B19), we can obtain

$$
\mathcal{R} \rightarrow \tilde{C}_{1}=\text { const. }
$$

From Einstein equations, the curvature perturbation is related with the scalar field fluctuation as

$$
\mathcal{R}=-\kappa_{4}^{2} \frac{\dot{\phi}}{2 H} \delta \phi .
$$

[1] R. Maartens, Living Rev. Rel. 7, 7 (2004) arXiv:gr-qc/0312059.

[2] L. Randall and R. Sundrum, Phys. Rev. Lett. 83, 4690 (1999).

[3] R. Maartens, D. Wands, B. A. Bassett and I. Heard, Phys. Rev. D 62, 041301 (2000) arXiv:hep-ph/9912464.

[4] K. Koyama, D. Langlois, R. Maartens and D. Wands, JCAP 0411 (2004) 002.

[5] K. Koyama, S. Mizuno and D. Wands, JCAP 0508, 009 (2005) arXiv:hep-th/0506102.

[6] A. D. Sakharov, Dokl. Akad. Nauk SSSR 177, 70, (1967) [Sov. Phys. Dokl.12, 1040 (1968)]; K. Akama, Y. Chikashige, T. Matsuki and H. Terazawa, Prog. Theor. Phys. 60, 868(1978); K. Akama, Prog. Theor. Phys. 60, 1900(1978); A. Zee, Phys. Rev. Lett. 42, 417 (1979); S. L. Adler, Phys. Rev. Lett. 44, 1567 (1980); S. L. Adler, Phys. Rev. Mod. Phys. 54, 729 (1982) [Erratum-ibid. 55, 837 (1983)].

[7] G. R. Dvali, G. Gabadadze and M. Porrati, Phys. Lett. B 485, 208 (2000) arXiv:hep-th/0005016.

[8] A. Lue, Phys. Rept. 423, 1 (2006) arXiv:astro-ph/0510068.

[9] M. Bouhmadi-Lopez, R. Maartens and D. Wands, Phys. Rev. D 70, 123519 (2004) arXiv:hep-th/0407162.

[10] E. Papantonopoulos and V. Zamarias, JCAP 0410, 001 (2004) arXiv:gr-qc/0403090.

[11] H. s. Zhang and R. G. Cai, JCAP 0408, 017 (2004) arXiv:hep-th/0403234.

[12] C. Deffayet, Phys. Lett. B 502, 199 (2001) arXiv:hep-th/0010186.

[13] M. A. Luty, M. Porrati and R. Rattazzi, JHEP 0309, 029 (2003) arXiv:hep-th/0303116.

[14] A. Nicolis and R. Rattazzi, JHEP 0406, 059 (2004) arXiv:hep-th/0404159.

[15] K. Koyama, Phys. Rev. D 72, 123511 (2005) arXiv:hep-th/0503191.

[16] D. Gorbunov, K. Koyama and S. Sibiryakov, Phys. Rev. D 73, 044016 (2006) arXiv:hep-th/0512097.

[17] C. Charmousis, R. Gregory, N. Kaloper and A. Padilla, arXiv:hep-th/0604086

[18] A. Lue and G. Starkman, Phys. Rev. D 67, 064002 (2003) arXiv:astro-ph/0212083; A. Lue, R. Scoccimarro and G. D. Starkman, Phys. Rev. D 69, 124015 (2004) arXiv:astro-ph/0401515 .

[19] K. Koyama and R. Maartens, JCAP 0601, 016 (2006) arXiv:astro-ph/0511634.

[20] N. Kaloper, Phys. Rev. D 71, 086003 (2005) [Erratum-ibid. D 71, 129905 (2005)] arXiv:hep-th/0502035; N. Kaloper, Phys. Rev. Lett. 94, 181601 (2005) [Erratum-ibid. 95, 059901 (2005)] arXiv:hep-th/0501028.

[21] S. Mukohyama, Phys. Rev. D 62, 084015 (2000).

[22] H. Kodama, A. Ishibashi and O. Seto, Phys. Rev. D 62, 064022 (2000).

[23] C. Deffayet, Phys. Rev. D 66, 103504 (2002) arXiv:hep-th/0205084.

[24] C. Deffayet, Phys. Rev. D 71, 103501 (2005) arXiv:gr-qc/0412114.

[25] J. Garriga and T. Tanaka, Phys. Rev. Lett. 84, (2000) 2778.

[26] T. Tanaka, Phys. Rev. D 69, 024001 (2004) arXiv:gr-qc/0305031.

[27] K. Koyama, JCAP 0409, 010 (2004) arXiv:astro-ph/0407263.

[28] K. Koyama and K. Koyama, Phys. Rev. D 72, 043511 (2005) arXiv:hep-th/0501232.

[29] H. van Dam and M. J. G. Veltman, Nucl. Phys. B 22, 397 (1970); V. I. Zakaharov, JETP. Lett. 12, 312 (1970); Y. Iwasaki, Phys. Rev. D 2, 2255 (1970).

[30] M. Fierz, Helv. Phys. Acta. 22, 3 (1939); M. Fierz and W. Pauli, Proc. R. Soc. London. A 173,211 (1939).

[31] M. Porrati, Phys. Lett. B 498, 92 (2001) arXiv:hep-th/0011152.

[32] A. Higuchi, Nucl. Phys. B 282, 397 (1987); A. Higuchi, Nucl. Phys. B 325, 745 (1989).

[33] I. I. Kogan, S. Mouslopoulos and A. Papazoglou, Phys. Lett. B 503, 173 (2001) arXiv:hep-th/0011138.

[34] U. Gen and M. Sasaki, Prog. Theor. Phys. 105, 591 (2001) arXiv:gr-qc/0011078.

[35] H. Yoshiguchi and K. Koyama, Phys. Rev. D 70, 043513 (2004) arXiv:hep-th/0403097.

[36] T. Shiromizu, K. i. Maeda and M. Sasaki, Phys. Rev. D 62, 024012 (2000).

[37] K. i. Maeda, S. Mizuno and T. Torii, Phys. Rev. D 68, 024033 (2003) arXiv:gr-qc/0303039.

[38] K. Koyama, A. Mennim and D. Wands, Phys. Rev. D 72, 064001 (2005) arXiv:hep-th/0504201.

[39] H. Yoshiguchi and K. Koyama, Phys. Rev. D 71 (2005) 043519 arXiv:hep-th/0411056.

[40] C. Deffayet, G. R. Dvali, G. Gabadadze and A. I. Vainshtein, Phys. Rev. D 65, 044026 (2002) arXiv:hep-th/0106001.

[41] A. Lue, Phys. Rev. D 66, 043509 (2002) arXiv:hep-th/0111168.

[42] A. Gruzinov, New Astron. 10, 311 (2005) arXiv:astro-ph/0112246.

[43] I. Giannakis and H. c. Ren, Phys. Lett. B 528, 133 (2002) arXiv:hep-th/0111127.

[44] M. Porrati, Phys. Lett. B 534, 209 (2002) arXiv:hep-th/0203014.

[45] D. K. Park, Phys. Lett. B 562, 316 (2003) arXiv:hep-th/0304056. 
[46] Y. Fujii and K. Maeda, The Scalar-Tensor Theory of Gravitation (Cambridge Univesity Press, Cambridge, UK, 2003)

[47] M. Sasaki, Prog. Theor. Phys. 76, 1036 (1986); V. F. Mukhanov, Sov. Phys. JETP 67, 1297 (1988) [Zh. Eksp. Teor. Fiz. 94N7, 1 (1988)]. V. F. Mukhanov, H. A. Feldman and R. H. Brandenberger, Phys. Rept. 215, 203 (1992). 